\title{
Study Smart Substation Condition Monitoring System Base on Internet of Things
}

\author{
Cheng Dawei \& Gao Qiang \& Geng Baohong \& Han Yue \& Li Yandan \\ The Electric Power Research Institute of Liaoning Province Power Company
}

\begin{abstract}
The substation equipment status monitoring system as an important means to ensure the safe operation of the electrical equipment as well as to enhance the level of production and operation management of substation equipment and which has become s hot spot for Smart grid researching. In this paper, Through the study of Smart Substation Condition Monitoring System propose a scheme for Smart substation condition monitoring system based on wireless sensors networks, Discussing the system architecture, solar power system, wireless communication module configuration problem, And the program is applied to 220kV Smart substation condition monitoring system, through run the system, as well as monitoring data analysis, That the design reduces the overall cost of construction and improve the performance of the system for the substation condition monitoring system and that has a very important significance for substation equipment condition monitoring system.
\end{abstract}

KEYWORD: Smart substation, Internet of things; Condition monitoring system; Interface Specifications of Smart Substation

\section{GENERAL INSTRUCTIONS}

Contents of this paper is based Liaoning Province Electric Power Co., China Electric Power Research Institute, Shenyang Institute of Automation and shared the major research topics MIIT "for the smart grid security monitoring, sensor network transmission efficiency, metering and user interaction development and application of verification." pilot projects in the field of substation. Based intelligent substation higher integration, more efficient, less area, the province invested more unified concept, condition monitoring system, a device for the first time the station will station level, process level all use a major national research projects with Chinese Ministry of autonomy wireless communication protocols of intellectual property, and to achieve IEC 61850 communication between the spacer layer and the station level via wireless technology, an alternative to traditional fiber optic communications. Project-based networking technology developed and photovoltaic power technology communications gateway machine, core / clamps ground current monitoring device, temperature measurement devices, surge arresters and SF6 insulation monitoring device density microwater monitoring devices, to achieve the things communications technology and condition monitoring truly integrated system to improve the reliability of Things communication. Meanwhile according to the integrated monitoring system construction specifications, standardization through I3 interface within the station integrated information platform, integrated information platform for centralized control center for data sharing;

\section{RELATED RESEARCH}

China Electric Power Research Institute and East China Power Grid Co., Ltd. is responsible for drafting the Q / GDW 534-2010 "substation equipment condition monitoring system Technical Guidelines" [1] provides a system architecture substation equipment condition monitoring system, monitoring system selection and configuration content principle, functional and technical requirements and other aspects. According to the State Grid Corporation of power transmission equipment condition monitoring system construction work requirements, on the basis of a large number of preliminary exploration of each unit, the company Production Technology Ministry State Grid Electric Power Research Institute, China Electric Power Research Institute and other units, research to develop a "power transmission equipment condition 
monitoring system level design. "[2] pointed out that the design of power transmission equipment condition monitoring system is an important means to achieve the status of operation of transmission and distribution equipment maintenance management, enhance the operation and management of power transmission specialized in producing lean levels. System through a variety of sensor technology, widearea communications technologies and information processing technology for real-time sensing of various types of transmission equipment operating status, monitoring and early warning, analysis and diagnosis and assessment predicted that its construction and extension work to improve the level of grid intelligence to achieve operation and management of power transmission equipment status has a positive and far-reaching significance. Q responsible for drafting the State Grid Electric Power Research Institute, China Electric Power Research Institute, NARI Technology Development Co., Ltd., etc. / GDW 678-2011 "intelligent substation integrated monitoring system functional specification" [3] and Q / GDW 679-2011 "intelligent substation integrated monitoring system construction technical specifications" [4], the former specifies the functional requirements of intelligent substation integrated monitoring system, a clear integration of intelligent substation monitoring system data collection, information transmission and interaction. The latter provides standardized architecture of intelligent substation monitoring system integration, system, network architecture, system configuration, data acquisition and transmission of information, security and other construction technical requirements. Things is the integration of sensor technology, information processing technology and network communication technology to achieve low cost, low-power, distributed, self-organizing features. Things wireless communication in substation automation field advantage it has great prospects, networking compared to existing substation automation system with high sampling accuracy, ease of laying, robust [5] and so on.

Section of this article reads as follows: Section II describes the framework of intelligent substation condition monitoring system, the station communication interface specification. Section III describes the structure of the monitoring system, solar power design, wireless networking module configuration state based on the content. Section IV describes the condition monitoring system of things of these research-based applications to He Jia Smart substation monitoring system construction, and part of the operating data for analysis.

\section{FRAMEWORK FOR INTELLIGENT SUBSTATION CONDITION MONITORING SYSTEM}

Intelligent substation monitoring system [6-7] design goal is strong and smart grid construction for the national grid ompany requirements, combined with "three five" development strategy, relying on the PMS, to establish a "two-tier deployment, three applications" in the company's range unified substation equipment condition monitoring systems, standardize various substation equipment condition monitoring data access, offers a variety of substation equipment condition monitoring information display, warning, analysis, diagnosis, assessment and forecasting capabilities, and focus on other relevant condition monitoring system provides data to achieve comprehensive monitoring substation equipment operation and management of the state and the state.

Overall, the master substation equipment condition monitoring system at the national grid company headquarters and provincial companies a complete two-stage deployment, the deployment of the station at the end substation monitoring unit, integrated monitoring unit and a variety of condition monitoring devices. Various types of substation equipment condition monitoring data in the network of provincial and corporate headquarters centralized storage.

According to the construction of thought within the State Grid Corporation of the station, the station into station control layer, the spacer layer and the process layer, at station level deployment station end monitoring unit, responsible for collecting the entire station status monitoring information, the spacer layer to deploy integrated monitoring unit, its role is monitoring means data collection is complete, and the data model mapped to IEC 61850, IEC 61850 and a communication protocol by the station side to the monitoring unit, the main installation process level monitoring device.

Condition monitoring through standardized data services at the provincial level condition monitoring data network, centralized storage via the Enterprise Service Bus (enterprise service bus, ESB) for other applications of various types of substation equipment condition monitoring data, meet all kinds of business applications on applications need to condition monitoring data. Substation equipment condition monitoring system provides relevant master data via data centers and other applications ESB share scheduler, lightning, weather and so on. Meanwhile, the network will focus on the provincial side PMS condition monitoring information (crossgrid section, together with cross-grid equipment and transport inspection information) further forwarding company headquartered side condition monitoring database through data centers to corporate 
headquarters in cross PMS power advanced application modules and other applications to use.

Condition monitoring system by the station level device (monitoring unit comprises a station side, the integrated application servers, data communication gateway, etc.), integrated monitoring unit, the monitoring device component, the system interface is schematically shown in Figure1.

Intelligent substation in condition monitoring systems, has standardized the integrated monitoring unit (or to satisfy DL / T 860 communication standard line monitoring systems) and communication protocols (ie, I1 interface specification) between the station level and station level equipment and state evaluation center master system time communication protocols (ie, I2 interface Specification). But still exist between the on-line monitoring devices and integrated monitoring unit agreement privatization, lack of information on-line monitoring device description model, resulting in data line monitoring device cannot be standardized access to integrated monitoring unit and how the condition monitoring system data and integrated information platform , centralized control center for data sharing and other issues. To solve the above problem and needs to standardize the communication interface protocol between the on-line monitoring devices and integrated monitoring unit (ie, I0 Interface Specification), standardized communication protocols intelligent substation monitoring system and monitoring systems, and other systems within the station (ie, I3 Interface Specification), condition monitoring system to achieve standardization of data communications.

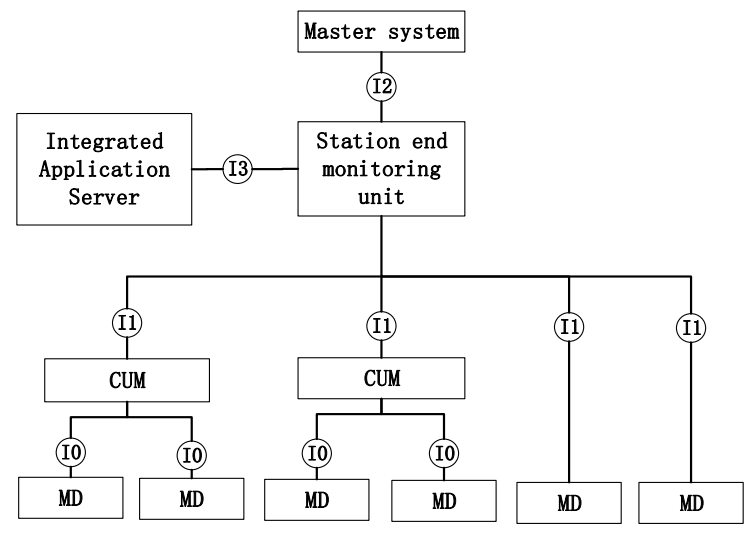

Fig. 1 Interface Status Monitoring System

\section{THINGS CONDITION MONITORING SYSTEM BASED ON}

There are very strong in the substation electromagnetic radiation, so the corona harm, the presence of electromagnetic interference on wireless communications cause some degree of interference.
However, in paper [8] stated that a corona discharge energy of a pulse current through the electromagnetic waves directly radiated, highfrequency interference caused by the surrounding environment, but after more than $20 \mathrm{MHz}$ frequency, the interference level decay rapidly, is almost zero.

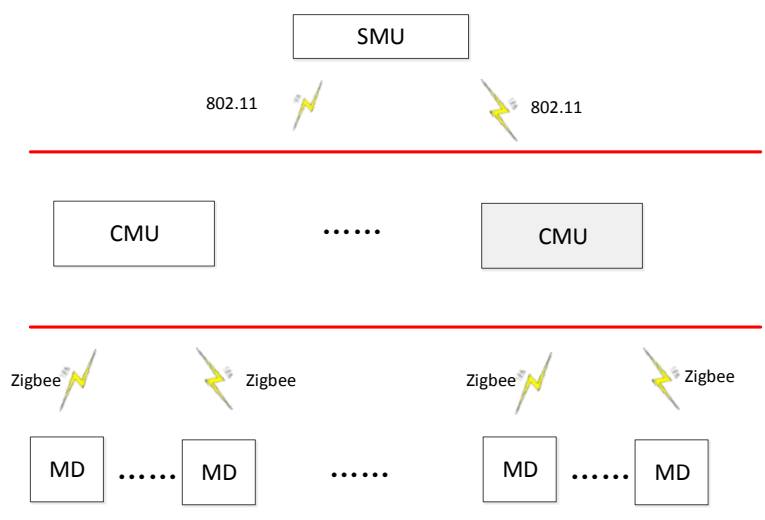

Fig.2 Things condition monitoring based on system configurtion diagram

Form Figure2, Integrated monitoring unit and the monitoring device process layer spacer layer composed star network communication using Zigbee standard protocol, select the desired wireless module can target existing RS485 bus (or RS232) developed wireless solutions to achieve RS485 bus (or transparent transmission RS232) and wireless communication between the RS485 bus can replace the existing (or RS232); integrated monitoring unit station terminal station level monitoring unit and a spacer layer of the same composition as a star network, communications protocol uses IEEE $802.11 \mathrm{~b} / \mathrm{g}$ standards.

\section{MONITORING SYSTEM OPERATIONAL ANALYSIS BASED ON THE STATE OF THINGS}

Lightning arrester condition monitoring one can improve the safe operation of early warning systems, early detection and troubleshooting, to avoid a major accident, on the other hand, can arrester leakage current and the number of operations performed statistical and data remote transmission, thereby effectively monitor area continuous operating conditions within the device, to better address the impact of contingency factors and unexpected situations, timely detection of arrester body moisture, component aging and other internal defects, eliminate hidden faults.

According Figure3, is a Ho-based Intelligent Substation $220 \mathrm{kV}$ arrester Things condition monitoring data collected once every half hour, and continuously collects 159 times. Released by the State Grid Corporation of specification [10] defines the full current normal range $<1.5$ times the 
measured value after the initial surge arresters installed resistive current is $<1.3$ times the initial measurement value arresters installed in the initial installation, all current and resistance current is maintained at about $0.42 \mathrm{~A}$ full current in the vicinity of $0.001 \mathrm{~A}$, the system is running, resistive current is maintained at about $0.02 \mathrm{~A}$. This gave the system is stable, condition monitoring systems meet the requirements of the arrester operation.

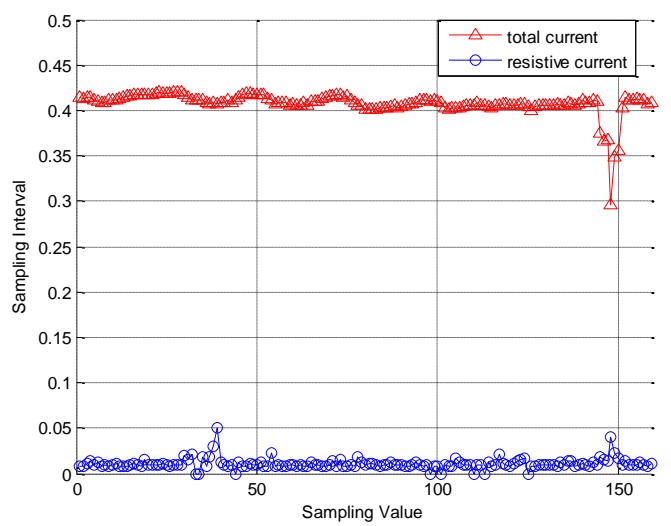

Fig. 3 Full current and surge arrester condition monitoring resistive current sampling distribution

Chromatography on-line monitoring of transformer oil is the use of fault features gas-line monitoring tools can overcome the traditional offline long experimental period, from sampling, transported to measure many links, tedious operation shortcomings, online continuous monitoring of gaseous components can provide a complete trend information on timely detection of potential failure, determine the transformer maintenance intervals, conduct life prediction.

\section{SUMMARY}

Things used in smart substation monitoring system has the advantage, however, is facing many difficulties, the transmission bandwidth, transmission distance, node power, electromagnetic compatibility and network security is a key problem. This paper analyzes the architecture of intelligent substation monitoring system as well as the state of things in the smart substation monitoring system application, according to the structural design of intelligent substation three hierarchical heterogeneous networking architecture and engineering are given for specific issues detailed solutions. Things condition monitoring system based on the design proposed in this paper have been deployed in Ho $220 \mathrm{kV}$ substation intelligent, Liaoning Province, and successfully put into operation.

\section{REFERENCES}

[1] Rahimi, F.; Ipakchi, A.; Demand Response as a Market Resource Under the Smart Grid Paradigm, IEEE Transactions on Smart Grid,Year: 2010 , Page(s): 82-88.

[2] Sooyeon Shin; Taekyoung Kwon; Gil-Yong Jo; Youngman Park; Rhy, H.; An Experimental Study of Hierarchical Intrusion Detection for Wireless Industrial Sensor Networks; IEEE Transactions on Industrial Informatics; Year: 2010,6(4),Page(s): 744-757.

[3] Buttyan, L.; Gessner, D.; Hessler, A.; Langendoerfer, P.; Application of wireless sensor networks in critical infrastructure protection: challenges and design options [Security and Privacy in Emerging Wireless Networks; IEEE Wireless Communications, 2010, 17(5); Page(s): 44 -49 .

[4] Weiwei Fang; Feng Liu; Fangnan Yang; Lei Shu; Nishio, S.;Energy-efficient cooperative communication for data transmission in wireless sensor networks; IEEE Transactions on Consumer Electronics; 2010,56(4); Page(s): 2185 -2192.

[5] Xue Wang; Junjie Ma; Sheng Wang; Daowei Bi; Distributed Energy Optimization for Target Tracking in Wireless Sensor Networks; IEEE Transactions on Mobile Computing, 2010,9(1); Page(s): 73-86.

[6] Ichihara, Y.; Challenges for engineers in the electric power industry; Power Engineering Review, 2000, 20(12), Page(s): 3-6

[7] Galli, S.; Scaglione, A.; Zhifang Wang; For the Grid and Through the Grid: The Role of Power Line Communications in the Smart Grid. Proceedings of the IEEE; 2011, 99(6); Page(s): 998 - 1027.

[8] P. Gupta and P. R. Kumar, "The capacity of wireless networks," IEEE Trans. Inform. Theory, vol. 46, no. 2, pp. 388-404, Mar. 2000.

[9] Theodore S. Rappaport. Wireless Communications: Principles and Practice (2nd Edition). Prentice Hall PTR, 2002:73-95.

[10] D. Ebehard and E. Voges, "Digital single sideband detection for interferometric sensors," presented at the 2nd Int. Conf. Optical Fiber Sensors, Stuttgart, Germany, 1984. 